\title{
Rhétorique des gwerziou
}

Rhetoric in the gwerziou

\section{Yves Le Berre}

\section{Q OpenEdition}

\section{Journals}

Édition électronique

URL : https://journals.openedition.org/lbl/1787

DOI : $10.4000 / \mathrm{lbl} .1787$

ISSN : 2727-9383

\section{Éditeur}

Université de Bretagne Occidentale - UBO

\section{Édition imprimée}

Date de publication : 1 février 2013

Pagination : 141-160

ISBN : 979-10-92331-00-4

ISSN : 1270-2412

\section{Référence électronique}

Yves Le Berre, "Rhétorique des gwerziou », La Bretagne Linguistique [En ligne], 17 | 2013, mis en ligne le 01 mai 2021, consulté le 22 mai 2021. URL : http://journals.openedition.org//bl/1787 ; DOI : https:// doi.org/10.4000/lbl.1787

\section{(c) (1)}

La Bretagne Linguistique est mise à disposition selon les termes de la Licence Creative Commons Attribution 4.0 International. 


\title{
Rhétorique des gwerziou
}

\begin{abstract}
C
hacun de nous est capable d'identifier spontanément de nombreuses formes littéraires alors qu'il n'en a lu ou entendu que quelques mots ou quelques phrases. Une tragédie classique, un poème lyrique, un conte merveilleux sont reconnus presque instantanément comme représentants d'un genre, et cette reconnaissance précoce dispose l'esprit à attendre que les règles de ce genre soient appliquées à l'ensemble du texte.

Il faut donc que nous possédions dans notre culture un système de codes qui, par le jeu des identités, des équivalences, des différences et des oppositions, nous permet d'identifier un texte à un ensemble d'autres textes similaires sans presque jamais nous tromper.

Pour le conte merveilleux, par exemple, eur wech e oa... 'il était une fois...' est un indicateur qui nous permet de régler d'emblée notre réceptivité sur l'ensemble des codes propres à ce genre.

J'avais dans mon jeune temps une tante originaire de Poullan (tout près de Douarnenez), et d'ailleurs nièce d'Yvon Crocq, auteur entre autres ouvrages des Marvailhou Kerne 'Contes de Cornouaille' publiés en 1910. Elle avait la réputation d'être une très bonne chanteuse de gwerziou ou complaintes en breton, qu'elle tenait de sa
\end{abstract}

* Professeur émérite de celtique, CRBC (EA 4451), UBO/ueb 
mère et de sa grand-mère. Aussi était-elle parfois invitée, à la belle saison, à passer la soirée dans les maisons du quartier; elle «payait» son vin chaud en interprétant une de ses gwerz. Il m'est arrivé de l'accompagner à ces soirées et, même si je ne comprenais pas toutes les paroles, je savais très bien qu'il s'agissait de chansons racontant des choses tristes : crimes et malheurs.

J'ai eu ensuite l'occasion d'étudier quelques gwerz avec des étudiants de licence ou de maîtrise et j'ai comme tout le monde été frappé par leur unité d'écriture, mais sans aller plus loin dans la recherche des traits spécifiques à l'origine de ce sentiment. Je voudrais aujourd'hui commencer à combler cette lacune en analysant les procédés d'écriture de trois gwerz prises au hasard dans les deux volumes publiés par Luzel en 1868 et 1874 : Ar Vinorezik '1'Orpheline', ci-dessous AV ; Margodig Gillard, ci-dessous MG ; Kervegan hag An Tourello, ci-dessous KT. Deux héroïnes : une matricide et une infanticide; deux héros : gentilshommes amoureux de la même femme, dont l'un mourra de la jalousie de l'autre.

Je n'examinerai pas ici les caractères formels communs aux gwerz, leur versification en particulier, qui ne permettent d'ailleurs pas de les distinguer d'autres genres proches, le cantique, par exemple. Je m'en tiendrai aux traits spécifiques récurrents de leur écriture, donc à leur arsenal rhétorique.

Nous nous en tiendrons pour l'instant à la définition courante de la rhétorique comme ensemble de procédés d'écriture visant à «améliorer la qualité de l'énoncé, en agrémenter la forme, en renforcer $l^{\prime}$ effet ${ }^{1} \gg$. La rhétorique serait donc l'ensemble des procédés conscients, sélectionnés dans un code socialement partagé, par lesquels l'auteur gère sa communication et sa relation avec le récepteur.

Quoique conventionnel, le trait rhétorique fait aspérité dans le texte dans la mesure où il capte autoritairement l'attention du lecteur, par opposition à l'expression sémantiquement équivalente, mais lisse, qui laisse l'auditeur plus libre d'accepter ou de refuser l'énoncé.

1. H. Bonnard, Code du français courant, Paris, Magnard, 1987, p. 40. 
Je me place pour l'instant dans l'attitude de celui qui écoute une chanteuse interpréter une gwerz et dont l'attention est attirée ici et là par des configurations plus ou moins remarquables de mots et de syntagmes $^{2}$, donc par des accidents dans le cours ordinaire ${ }^{3}$ du récit.

Je vais relever dans les trois textes reproduits ci-dessous tout ce qui, subjectivement, me semble inattendu, ce qui interrompt le déroulement régulier du récit, ce qui concourt à en rendre le registre plus paritaire ou plus disparitaire dans les 256 vers du corpus. Je répartirai ensuite les accidents en catégories homogènes, mesurerai leur présence relative dans les trois textes et essaierai de comprendre à quelle fin chacun d'entre eux est utilisé (partie 1). Ayant ensuite regroupé tous les types d'accident et leurs occurrences estimées sur un tableau, je tâcherai de voir s'ils entretiennent entre eux des relations de similarité, d'opposition, de complémentarité qui, à défaut de les constituer en système ${ }^{4}$, leur donnent au moins une certaine cohérence (partie 2). Il sera alors peut-être temps de revenir sur mes propos initiaux et de tâcher de comprendre comment, dans les gwerz, s'articulent les lois du genre, les caractères modaux, les manifestations du style et les «tours» de la rhétorique (partie 3).

\section{Description}

\section{La phrase à sujet initial}

Alors que dans la langue orale le sujet placé devant le verbe est statistiquement assez rare ou de nature dialectale (hors Trégor et Léon), les gwerz le mettent très souvent en tête de phrase, même là où il n'est pas le prédicat.

2. Comme l'idée principale est de définir ce que pourrait être une rhétorique de la gwerz, je laisse de côté tout ce qui concerne le rythme et les sons.

3. Par cours ordinaire, j'entends un état (théorique) du discours dans lequel la forme se fait complètement oublier au profit du contenu narratif. Dans les Exercices de style de Queneau, c'est exactement le contraire : le contenu narratif n'est qu'un prétexte à faire varier la forme, qui retient toute l'attention.

4. Comme ils font partie d'un ensemble non fini, je ne crois pas possible qu'ils forment une structure. 


\begin{tabular}{|lcccc|}
\hline \multirow{3}{*}{ Sujet en tête } & AV & MG & KT & T. \\
& 17 & 11 & 19 & 47 \\
& $18,89 \%$ & $15,28 \%$ & $29,69 \%$ & \\
\hline
\end{tabular}

La phrase - ou la proposition principale de la phrase - à sujet initial représente près de $20 \%$ du total des accidents recensés. Dans les autres phrases, c'est le verbe ou un syntagme circonstanciel qui commence la phrase. Les chiffres bruts donnés ci-dessus cachent une réalité plus tranchée. KT en est de loin le plus grand utilisateur : la phrase à sujet initial, qui y apparaît toutes les 5 lignes en moyenne, représente près du tiers de son total d'accidents (19/64).

Elle se rencontre tellement souvent dans les trois textes qu'elle apparaît comme un véritable procédé technique de «traction» du récit. Elle confère en outre à ce dernier un rythme solennel et un peu monotone accordé à la gravité du thème.

\section{Les apostrophes}

Alors que dans le récit ordinaire les protagonistes sont généralement désignés par un nom ou un pronom de la $3^{\mathrm{e}}$ personne, dans les gwerz apparaissent souvent des phrases au style direct dans lesquelles un personnage s'adresse à un autre par son nom, par un pronom ou par un impératif.

\begin{tabular}{|ccccc|}
\hline \multirow{3}{*}{ Apostrophes } & AV & MG & KT & T. \\
& 16 & 11 & 12 & 39 \\
& $17,78 \%$ & $15,28 \%$ & $18,75 \%$ & \\
\hline
\end{tabular}

Second accident en fréquence, l'apostrophe s'insère dans un cadre discursif globalement stable (1 ère personne quand il s'agit d'une confession, 3 e personne quand il s'agit d'une narration); point culminant de petits dialogues à l'intérieur du récit, elle théâtralise celui-ci. Elle est une sorte de delco produisant une étincelle d'énergie au début d'un passage au style direct, pour le lancer. Elle compense ainsi la lourdeur que les séries de phrases SVO, de reprises et d'annonces introduisent forcément dans le déroulement du récit. 


\section{Les reprises de vers}

Ce procédé consiste a. à répéter exactement le même vers, soit immédiatement, soit un peu plus bas dans le texte; b. à répéter seulement une partie du vers; c. à reprendre les mots d'un vers dans un autre vers, mais dans un ordre différent.

\begin{tabular}{|ccccc|}
\hline \multirow{3}{*}{ Reprises } & AV & MG & KT & T. \\
& 14 & 10 & 11 & 35 \\
& $15,56 \%$ & $13,89 \%$ & $17,19 \%$ & \\
\hline
\end{tabular}

La reprise, proportionnellement répartie à peu près également dans les trois textes (entre 14 et 17\%), est sans doute de ce fait, avec l'apostrophe, la figure la mieux intégrée à la forme " gwerz».

Les reprises cousent ensemble deux vers d'une même strophe ou de deux (voire trois) strophes successives. Elles ralentissent le cours du récit. Elles donnent de l'importance à l'élément répété. Elles contribuent à allonger les séquences de rimes identiques. Globalement, elles dramatisent par insistance et étirement du temps.

\section{Les appellatifs «affectifs" : hypocoristique, qualificatif}

Les noms désignant des catégories sociales sont souvent flanqués du suffixe -ig et/ou d'un qualificatif ou d'un syntagme qualificatif.

\begin{tabular}{|ccccc|}
\hline \multirow{3}{*}{ Affectivité } & AV & MG & KT & T. \\
& 11 & 10 & 1 & 22 \\
& $12,22 \%$ & $13,89 \%$ & $1,56 \%$ & \\
\hline
\end{tabular}

L'appel à la compassion par adjonction au nom de l'hypocoristique -ig ou de qualificatifs ( $\mathrm{mad}$, paour) est bien plus fréquent dans $\mathrm{AV}$ et $\mathrm{MG}^{5}$ que dans $\mathrm{KT}$ où seule la marquise en bénéficie, alors que la principale victime est son frère Des Tourelles.

5. Je ne comprends pas le sens du menig rouz de 16.3. et du tammig teill de 19.2., et ne les compte donc pas comme accidents, quoiqu'ils me semblent inattendus. Menig rouz peut être un poncif ancien : le v. 38 du lai du Laüstic (Marie de France) dit que rien ne séparait les deux amants «fors un haut mur de piere bise». 
On trouve dans AV bugelik (1) et mestrik (28, 33, 35), mais pas mestrezik; cette dernière est toujours qualifiée de mestrez (17, $22,23,30,42)$. L'utilisation du «diminutif» est donc purement hypocoristique et interdite envers quelqu'un à qui on va faire, on fait ou on a fait du mal. En revanche, mad est une indication objective, qui peut se cumuler (ou non cf. 11,35) avec le diminutif : mestrik mad $(28,33)$. Cela dit, il est intéressant de constater que son emploi commence dès le 11.1 pour le père, et seulement au 17.2 pour la mère (au moment même du meurtre!). Les parents adoptifs, eux, ne la nomment jamais que minorez. Le premier -ig en appelle évidemment à la pitié de l'auditeur pour la pauvre orpheline; les seconds (renforcés par $\mathrm{mad}$ ) indiquent l'affection particulière que porte la récitante à son père adoptif; l'absence des troisièmes marque une absence d'affection pour la mère, malgré la bonté objective du référent. La distribution des - ig constitue donc deux couples de personnages (me-mestrig vs me-mestrez) dont les relations, dès la première partie du chant, indiquent implicitement la situation de déséquilibre qui va provoquer le drame. Dans MG il est frappant que l'infanticide soit toujours nommée par un double hypocoristique (Margod + ig), jamais par son prénom officiel (Marc'harid), comme si l'auteur et son public la considéraient davantage comme une victime que comme une criminelle. Intéressant aussi que la série des merc'hedigo s'interrompe soudain après la malédiction, comme si la condamnée prenait alors ses distances avec les vivants.

\section{Les symétries et symétries inversées}

La symétrie met en évidence : a. deux mots différents (miz/bloaz) insérés dans le même contexte à deux endroits différents; $b$. une paire de propositions, l'une à la forme affirmative ou interrogative, l'autre à la forme négative; c. la syntaxe identique de deux phrases composées avec des mots différents; $d$. des mots de valeurs opposées placés dans des positions où ils semblent se répondre.

\begin{tabular}{|ccccc|}
\hline \multirow{3}{*}{ Symétries } & AV & MG & KT & T. \\
& 6 & 10 & 5 & 21 \\
& $6,67 \%$ & $13,89 \%$ & $7,81 \%$ & \\
\hline
\end{tabular}


MG en est de loin le texte le mieux pourvu.

La symétrie crée dans le récit un balancement interne qui dédouble les éléments qui la forment, suggérant le sentiment d'une opposition structurelle entre le bien et le mal, entre le bonheur et le malheur, entre la vie et la mort, et qui renforce l'effet tragique provoqué par ailleurs par les sujets initiaux et les reprises : la minorez obéit et tue; Margodig est certes belle, mais elle n'est pas un bon exemple; Des Tourelles monte sur son cheval, puis en descend pour mourir.

\section{Les annonces}

Elles désignent le personnage qui va prendre la parole au style direct dans le récit. L'interprète dit quelque chose comme : «Alors X dit à $\mathrm{Y}: \ldots »$.

\begin{tabular}{|ccccc|}
\hline \multirow{3}{*}{ Annonces } & AV & MG & KT & T. \\
& 7 & 2 & 11 & 20 \\
& $7,78 \%$ & $2,78 \%$ & $17,19 \%$ & \\
\hline
\end{tabular}

Figure presque obligée de l'oralité, puisqu'il faut bien dire aux auditeurs quel personnage du récit va s'exprimer au style direct et à qui il va s'adresser, l'annonce de prise de parole, rare dans $\mathrm{MG}$, un peu plus répandue dans $\mathrm{AV}$, produit en outre dans KT un effet d'accumulation d'autant plus fort que presque toutes ces formules y suivent l'ordre SVO (ce qui n'est pas le cas dans AV).

Souvent précédée d'une ellipse temporelle, elle découpe la narration en "plans» successifs de la même façon que les «et $\mathrm{p}(\mathrm{u}) \mathrm{is} . . . »$ dans les récits enfantins.

\section{Les nombres fatidiques}

En des temps où peu de gens savent manipuler les chiffres, l'énonciation de nombres précis revêt une importance symbolique qu'elle n'a plus pour nous, aujourd'hui ${ }^{6}$.

6. Voir le «septante fois sept fois» de l'Évangile (Matthieu 18:22), qui signifie, non pas 490, mais 'autant de fois qu'il le faudra'. 


\begin{tabular}{|ccccc|}
\hline \multirow{3}{*}{ Nombres } & AV & MG & KT & T. \\
& 7 & 4 & 3 & 14 \\
& $7,78 \%$ & $5,56 \%$ & $4,69 \%$ & \\
\hline
\end{tabular}

Le plus remarquable n'est pas tant la fréquence que la quasiunanimité des trois textes à utiliser les nombres 3,7 et 18 , comme s'ils avaient une valeur poétique particulière. Ces nombres, surtout quand ils sont répétés, ont assurément une signification symbolique ou anthropologique.

Ainsi dans AV, «dix-huit» : l'habit neuf est le premier vêtement de la jeune fille sortant de l'enfance (rite de passage à sept ans dit 'âge de raison'). Quand elle a vingt-trois ans (10.2.), elle est chez eux depuis dix-huit ans. Elle était donc dans sa sixième année quand elle y est arrivée. Dix-huit mois plus tard, elle va avoir ou elle vient d'avoir sept ans. Le nombre 7 a aussi une valeur rituelle : sept vertus cardinales et théologales, sept péchés capitaux, sept sacrements etc. Enfin les 3 évanouissements et les 3 rétablissements du jeune veuf renvoient, entre autres choses, aux Évangiles: Pierre a renié Jésus trois fois, Jésus est tombé trois fois etc.

Dans MG l'héroïne a dix-huit ans; elle n'a plus trois jours à vivre. Ce n'est pas ici la mise en symétrie, mais le contraste entre les deux nombres qui leur donne du poids. Les trois enfants morts sous la pierre de la prairie ${ }^{7}$, les sept livres de pierre : ce sont les mêmes nombres que dans $\mathrm{AV}$, mais cette fois ils ne sont pas utilisés par couples. Les sept livres correspondent à peu près au poids nécessaire pour maintenir le corps d'un nouveau-né sous l'eau ${ }^{8}$.

Dans KT les «deux ou trois demoiselles de compagnie» ne me paraissent pas avoir de valeur " fatidique ${ }^{9} »$, contrairement aux sept (deux fois) et trois messes. Quelles que soient les explications techniques, historiques ou anthropologiques justifiant l'apparition de ces nombres dans les textes, on ne peut que remarquer la constance de 3,7 et 18. Ce qui laisse soupçonner plutôt un souci conventionnel d'ornementation «hiératique».

7. Plus un dans le lavoir, un dans le puits et un sous le seuil, ce qui fait 6 répartis dans tout l'espace de l'intimité familiale.

8. 3,2 kg en moyenne aujourd'hui, mais ils devaient être plus petits à l'époque.

9. Il s'agit simplement de montrer que c'est une jeune fille de haut rang social. 


\section{L'utilisation du participe présent comme verbe principal}

Là où l'on attendrait un verbe fini (terminé par les marques du mode, du temps, de la personne), la phrase est construite autour d'un verbe non fini qui apparait sous la forme du groupe o+verbe-nom, dont la fonction ordinaire est la qualification.

\begin{tabular}{|ccccc|}
\hline \multirow{3}{*}{ Gérondives } & AV & MG & KT & T. \\
& 7 & 0 & 0 & 7 \\
& $7,78 \%$ & $0,00 \%$ & $0,00 \%$ & \\
\hline
\end{tabular}

La phrase narrative à verbe au participe présent est une particularité de AV. Elle me semble indiquer que l'événement rapporté sous cette forme est plutôt inattendu pour la narratrice. Elle marque en outre les tournants du récit. Enfin, construite autour d'une forme verbale non finie, elle distend le lien étroit qui unit ordinairement le sujet et son verbe : le sujet subit en quelque sorte davantage l'événement qu'il ne l'accomplit.

\section{L'exclamation pathétique}

L'énonciateur exprime une vive émotion et somme l'auditoire de la partager.

\begin{tabular}{|ccccc|}
\hline & AV & MG & KT & T. \\
Pathétique & 1 & 3 & 1 & 5 \\
& $1,11 \%$ & $4,17 \%$ & $1,56 \%$ & \\
\hline
\end{tabular}

Ces cinq formules ont un caractère métalinguistique : l'auteur ou le personnage souligne ainsi, en s'adressant directement à son auditoire, la force exceptionnelle du sentiment ressenti.

\section{Les formulations " animistes"}

Ces formulations reposent sur la croyance en une vie dans un autre monde (pas forcément chrétien). Des morts, voire des êtres non identifiés, peuvent intervenir physiquement dans le monde des vivants. 


\begin{tabular}{|ccccc|}
\hline & AV & MG & KT & T. \\
Animisme & 3 & 2 & 0 & 5 \\
& $3,33 \%$ & $2,78 \%$ & $0,00 \%$ & \\
\hline
\end{tabular}

Seul KT ne fait aucune allusion à un être métaphysique.

Les morts qui réclament justice (la mestrez vad et l'inosant du puits) font normalement partie du fantastique chrétien ${ }^{10}$. La « chose» de AV est bien plus mystérieuse, que ce soit la voix d'un esprit de l'autre monde (interprétation traditionnelle) ou celle de l'inconscient de la minorez (interprétation moderne). Ce qui est certain, c'est que son intervention s'allie aux phrases à verbe participial pour retirer autant que faire se peut à la minorez le statut de personnage agissant de son plein gré, en pleine conscience de ce qu'elle fait.

\section{Les imprécations}

Le prédicat du récit rapporté par la gwerz étant généralement un méfait, le coupable de ce méfait encourt la vindicte publique et une punition. L'auteur du texte, en le maudissant par la voix de sa victime (AV, MG) ou par celle des honnêtes gens (KT), exige fortement sa condamnation.

\begin{tabular}{|ccccc|}
\hline \multirow{3}{*}{ Imprécations } & $\mathrm{AV}$ & $\mathrm{MG}$ & $\mathrm{KT}$ & $\mathrm{T}$. \\
& 1 & 1 & 1 & 3 \\
& $1,11 \%$ & $1,39 \%$ & $1,56 \%$ & \\
\hline
\end{tabular}

L'imprécation, comme il est logique, apparaît une fois dans chaque texte, vouant chacun des trois coupables aux rigueurs de la justice humaine $(\mathrm{AV}, \mathrm{KT})$ ou transcendante $(\mathrm{MG})$. Dans $\mathrm{MG}$, c'est à partir de cette phrase que Margodig bascule du statut de meurtrière à celui de victime. Ses deux fautes (les grossesses illégitimes répétées et la non-dénonciation des crimes du clerc) ne sont pas passibles de la peine de mort. Elle va donc mourir à la place du véritable coupable, ce qui mérite bien une petite malédiction. Et du coup elle peut être

10. Comme la statue du Commandeur, père de Doña Anna, dans Don Juan. 
l'objet de notre compassion (les hypocoristiques répétés). KT se situe dans le cadre de la justice humaine institutionnelle. Le duel ne s'est pas déroulé à la loyale et relève par conséquent de l'assassinat, d'un acte non noble (ignoble); la vie de Kervégan paiera donc pour la mort de Des Tourelles.

\section{Les métaphores}

La métaphore, procédé par excellence de la rhétorique classique et ecclésiastique, se distingue de la comparaison par l'absence d'agent de liaison ('fort comme un Turc').

\begin{tabular}{|ccccc|}
\hline \multirow{4}{*}{ Métaphores } & AV & MG & KT & T. \\
& 0 & 2 & 0 & 2 \\
& $0,00 \%$ & $2,78 \%$ & $0,00 \%$ & \\
\hline
\end{tabular}

Elle n'apparaît ici que dans MG : elle est double et répétée trois fois, comme si son auteur la trouvait particulièrement élégante. La fleur de lys symbolise la pureté; c'est un attribut courant de la Vierge Marie dans la poésie médiévale. Le «miroir» est ici le modèle de beauté; Miroir des dames ou Miroir aux dames est le titre de plusieurs poèmes célèbres du $\mathrm{XV}^{\mathrm{e}}$ siècle.

\section{L'anticipation}

Normalement un événement objectif intervient; ensuite seulement il provoque une réaction de l'intéressé. Ici, c'est l'inverse.

\begin{tabular}{|ccccc|}
\hline \multirow{3}{*}{ Anticipation } & AV & MG & KT & T. \\
& 0 & 1 & 0 & 1 \\
& $0,00 \%$ & $1,39 \%$ & $0,00 \%$ & \\
\hline
\end{tabular}

L'anticipation n'apparaît qu'une fois dans le corpus, encore dans MG. Sa fonction est, au sens propre, surréaliste : le contenu de la lettre provoque écoulement de larmes et saignement de nez avant même d'avoir été lu, comme s'il sautait à la figure de Margodig sans passer par sa conscience. Ce procédé est d'une très grande force 
rhétorique. La parole populaire a des formules proches : «il pleure avant d'avoir mal»; «je n'avais pas ouvert la bouche qu'il me traitait déjà de menteur »...

\section{Les jeux d'énonciation}

Dans Le Cid Don Diègue (père du Cid) et le comte (père de Chimène) se vouvoient, selon l'usage des courtisans, jusqu'au moment où ils se défient à l'épée. Ils passent alors au tutoiement viril. Lié au mode tragique, cet accident est typiquement rhétorique ${ }^{11}$. Avant de déterminer si nos textes contiennent des accidents d'énonciation, il faut d'abord déterminer la règle générale.

Celle-ci semble être sexuée : les hommes se tutoient entre eux et vouvoient les femmes. Les femmes n'utilisent que le «vous».

\begin{tabular}{|ccccc|}
\hline \multirow{3}{*}{ Énonciation } & $\mathrm{AV}$ & $\mathrm{MG}$ & $\mathrm{KT}$ & $\mathrm{T}$. \\
& 0 & 1 & 0 & 1 \\
& $0,00 \%$ & $1,39 \%$ & $0,00 \%$ & \\
\hline
\end{tabular}

Une seule infraction (dans MG) aux règles générales de l'énonciation (11.2. Mill mallos did, kloarek ingrat), liée à l'imprécation qui s'en trouve renforcée et paradoxalement ${ }^{12}$ solennisée. Dans le registre de l'imprécation, très «littéraire», le tutoiement est une manière traditionnelle de rabaisser le destinataire de la malédiction, surtout adressé par une femme à un homme.

\section{Distribution}

Il existe ainsi de notables différences entre les trois textes dans l'usage qu'ils font des procédés rhétoriques.

11. Enfin, c'est moi qui le dis... Les rhétoriciens prétendent que le procédé relève de l'énallage; les grammairiens assurent que ce n'est pas une figure de style!

12. Paradoxalement, puisqu'il s'agit d'un passage au paritaire, comme dans la tragédie classique. 


\begin{tabular}{|llrrr|r|r|}
\hline & & AV & MG & KT & T. & \\
\hline 1 & Sujet en tête & 17 & 11 & 19 & 47 & $20,80 \%$ \\
2 & Apostrophes & 16 & 11 & 12 & 39 & $17,26 \%$ \\
3 & Reprises & 14 & 10 & 11 & 35 & $15,49 \%$ \\
4 & Affectivité & 11 & 10 & 1 & 22 & $9,73 \%$ \\
5 & Symétries & 6 & 10 & 5 & 21 & $9,29 \%$ \\
6 & Annonces & 7 & 2 & 11 & 20 & $8,85 \%$ \\
7 & Nombres & 7 & 4 & 3 & 14 & $6,19 \%$ \\
8 & Gérondives & 7 & 0 & 0 & 7 & $3,10 \%$ \\
12 & Métaphores & 0 & 6 & 0 & 6 & $2,65 \%$ \\
9 & Pathétique & 1 & 3 & 1 & 5 & $2,21 \%$ \\
10 & Animisme & 3 & 2 & 0 & 5 & $2,21 \%$ \\
11 & Imprécations & 1 & 1 & 1 & 3 & $1,33 \%$ \\
13 & Anticipation & 0 & 1 & 0 & 1 & $0,44 \%$ \\
14 & Enonciation & 0 & 1 & 0 & 1 & $0,44 \%$ \\
\hline \multicolumn{2}{|l}{ Totaux } & 90 & 72 & 64 & 226 & $100,00 \%$ \\
\hline
\end{tabular}

La colonne T. donne le total des occurrences d'un même accident dans l'ensemble du corpus. La colonne de droite donne le pourcentage des occurrences de cet accident relativement au total des occurrences de tous les accidents du corpus (226). L'avant-dernière ligne rappelle le nombre de lignes de chaque texte. La dernière ligne donne le rapport entre le nombre de lignes d'un texte et le nombre d'accidents relevés dans ce texte.

Le plus long des trois, AV (94 lignes) est aussi celui qui cumule le plus grand nombre d'accidents : 90, soit presque un par ligne ${ }^{13}$, en moyenne. KT, qui n'est qu'un peu plus court (86 lignes), en contient nettement moins : 64 (3 pour 4 lignes). Enfin MG, le plus court des trois (76 lignes), en a 72 (presque un par ligne, comme AV). Malgré cette disparité, le poids de la rhétorique est donc globalement très important dans le corpus étudié.

13. Ce n'est qu'un ordre de grandeur : certaines lignes contiennent en effet plusieurs incidents rhétoriques. 
Aucun texte ne fait usage de tous les procédés identifiés. AV et MG, les plus «riches», en utilisent respectivement 11 sur 14 et 13 sur 14 . KT, le plus facile à dater de l'époque moderne ${ }^{14}$, en utilise seulement 9 , majoritairement groupés dans les trois premières lignes du tableau (42 occurrences sur 64).

Les trois premières lignes représentent 121 accidents sur 226, soit près de $54 \%$ du total. Elles contiennent probablement les formules favorites des auteurs de gwerz (sous réserve de sondages complémentaires) et, pour les auditeurs, les formules les plus typiques du genre «gwerz».

Les formules des trois lignes suivantes sont moitié moins nombreuses $(63$, soit $28 \%$ du total).

Les $18 \%$ restants (42 occurrences au total) sont répartis sur huit types différents d'accidents qui n'apparaissent qu'en petit nombre et sont souvent tout à fait absents de l'un ou de deux des trois textes.

\section{Interprétation}

Ces quatorze catégories d'accidents, dont toutes les occurrences font saillie dans le discours, me paraissent tirer leur énergie particulière de deux ressorts différents : l'accumulation et l'infraction.

\section{L'accumulation}

La phrase à sujet initial, l'apostrophe, la reprise de vers, la symétrie, l'annonce, les nombres fatidiques et la phrase participiale utilisent toutes la répétition, l'isotopie (intratextualité), la référence à du «déjà dit» (intertextualité). Si elles surprennent, c'est par l'itération du même.

Ces sept formules (183 occurrences, $80 \%$ du total) sont techniquement les plus primitives : on est là dans l'archéologie du récit oral.

14. Primitivement le marquisat était un commandement militaire important s'exerçant sur un vaste territoire frontalier. Sous l'Ancien Régime, c'est simplement un titre accordé aux possesseurs de fiefs assez importants, composés de trois baronnies et d'au moins trois châtellenies ou de deux baronnies et de six châtellenies. 


\section{L'infraction}

L'appel à l'affectivité de l'auditeur, les exclamations pathétiques constituent des violations de la règle tacite d'objectivité du récit ou à la neutralité du récitant, donc du principe de vraisemblance ${ }^{15}$.

Les interventions de l'autre-monde, les métaphores et l'anticipation constituent des altérations du fonctionnement ordinaire du monde réel : les morts n'ont plus de vie propre, une jeune fille n'est pas une fleur, l'effet ne peut précéder la cause.

Les imprécations sont une usurpation du rôle de la divinité ou de l'institution judiciaire, une façon de leur forcer la main : la mestrez n'a pas à prononcer la peine encourue pour son propre meurtre; Margodig n'est pas en position de punir elle-même le clerc ; Lokrenan ne peut préjuger du verdict du tribunal qui jugera Kervegan.

Le tutoiement anomal est une dérogation à la règle sociolinguistique générale qui veut que les femmes vouvoient les hommes.

Ces sept formules (43 occurrences, $20 \%$ du total) ne fonctionnent pas de façon aussi mécanique que les précédentes : elles supposent pour être comprises que l'auditoire maîtrise des prérequis de nature culturelle, particulièrement le fait que tout texte est une recomposition artificielle de la réalité.

Nos gwerz contiendraient donc deux strates d'accidents présents en nombre proportionnel à leur ancienneté, inversement proportionnel à leur complexité. Une strate remontant à la tradition orale (3.1.) et une autre liée davantage à la sphère de la lecture-écriture (3.2.). L'hypothèse qui sous-tend cette appréciation repose sur la conviction qu'il est plus facile de chanter deux fois de suite la même phrase ou de faire répondre à une phrase interrogative par sa semblable affirmative ou négative que de trouver une belle métaphore ou d'obliger l'auditeur à ressentir de la compassion pour une criminelle. Je ne prétends évidemment pas que la littérature orale ancienne aurait ignoré la métaphore, l'imprécation ou l'appel à la pitié. Seulement il me semble probable que qualifier Margodig Gillard précisément de

15. Je croirai davantage à la véracité d'un récit dont le narrateur est émotionnellement neutre. 
«fleur de lys des jeunes filles» renvoie à la littérature mariale médiévale et que la nommer «miroir des dames » fait référence aux poèmes $\mathrm{du} \mathrm{XV}^{\mathrm{e}}$ siècle portant ce titre.

Cela plaiderait en faveur de l'hypothèse d'une origine ancienne du genre «complainte», mais pas forcément des récits eux-mêmes dans la forme sous laquelle ils ont été recueillis par Luzel. Les lais de Marie de France, composés au XII siècle, ont une rhétorique toute différente. En revanche la gwerz Le Rossignol ${ }^{16}$, qui reprend l'argument du Lai du Laüstic, contient les mêmes procédés que nos trois complaintes. Marie de France déclare sans ambiguïté qu'elle a écrit en s'inspirant d'une chanson bretonne de son temps ${ }^{17}$. Il ne faut donc pas confondre le thème lui-même - assurément antérieur au XII ${ }^{\mathrm{e}}$ siècle - et sa mise en forme poétique, fondée sur l'accumulation de procédés oraux traditionnels ou écrits littéraires et très probablement postérieure (en raison de la présence de ces procédés d'origine littéraire).

Mon hypothèse est que le genre breton de la complainte, caractérisé par ses thèmes tragiques et sa rhétorique composite, s'est fixé dans sa forme moderne à une époque où la culture orale était encore très prégnante dans le milieu social décrit (petite noblesse rurale ${ }^{18}$ ), mais où la culture écrite avait déjà commencé aussi à se répandre. Donc, pour ce qu'on en sait, entre le XVe et le XVII ${ }^{\mathrm{e}}$ siècle.

16. Barzaz Breiz, p. 151 de l'édition de 1867.

17. «Un lai en firent li bretun : Le laüstic l'apelë hum.»

18. Les parents adoptifs de la Minorez veulent lui donner en dot une noblans 'terre noble'; Margodik Gillard est «le miroir des itronezed, 'dames'»; Kervégan et Des Tourelles sont deux gentilshommes. La langue que les personnages utilisent dans les passages en style direct est très mondaine : "Kaera noblanz a zo er vro; un darn, itron, euz hon mado»; «Em retiret, merc'hed iaouank...»; «'Welet 'r varkizes, leun a ganvo, o vriata ar c'horf maro». 


\section{Ar Vinorezik (Gwerziou I-106)}

1.1. Me oa 'r bugelik iaouank-flamm,

1.2. Pa varwaz ma zad ha ma mamm ;

2.1. Oblijet oann da glask ma boed,

2.2. Kapabl d'hen gonit na oann ket.

3.1. Ma oann leusket war ann hent-braz, 3.2. War ann hent-braz ewit enn glask.

4.1. Pa oann diskennet en hent doon,

4.2. Me o rankontr tud-a-feson ;

5.1. O rankontr aotro hag itron,

5.2. Pa oann diskennet [en] hent doon ;

6.1. Ma laraz 'nn aotro d'ann itron:

6.2. - Sell aze 'r bugel-a-feson ;

7.1. Hi c'homerromp ganimb ' $n$ hon zi,

7.2. 'Vel d'hon bugel greomp d'ez-hi.

8.1. Pa oann bet tric'houec' $h$ miz ' $n$ ho zi,

8.2. Oa gret un habit newez d'in :

9.1. Me a oa maget ha gwisket,

9.2. 'Vel pep-hini ann-he bewet.

10.1. P'oann bet tric'houec'h vloaz ' $n$ ho zi, 10.2. (Tri bloaz war-n-ugent ' $m$ boa neuze)

11.1. Laraz ma mestr mad d'am mestrez :

11.2. - Poent e dimizi 'r vinorez,

12.1. - Rei d'ei noblanz ar Feunteuniou,

12.2. Kaera noblanz a zo er vro ;

13.1. Kaera noblanz a zo er vro,

13.2. Un darn, itron, euz hon mado.

14.1. Ma laraz neuze ma mestrez:

14.2. - N' vo ket dimet ar vinorez ;

15.1. N' vo ket dimet ar vinorez,

15.2. Ken 'vo bet ganimb 'n pardon Agnez ;

16.1. Bep-bloa 'teu ganimb d'ar pardon,

16.2. Dre m'eo ur bugel-a-feson...
17.1. Pa arruaz ebars ar c'hoad,

17.2. 'Teu c'hoant-kousket d'am mestrez-vad;

18.1. Me 'c'h azeza war ar c'hlazenn,

18.2. Tapout hi fenn war ma barlenn ;

19.1. Tapout hi fenn war ma barlenn,

19.2. Hag a vanaz kousket soudenn.

20.1. O tont un dra da laret d'in :

20.2. - Laz da vestrez, zent a-ouz-in ;

21.1. Laz da vestrez, zent a-ouz-in,

21.2. Hag itron en hi flaz e vi !

22.1. Euz ann dra-ze am euz zentet,

22.2. Ma mestrez vad am euz lazet ;

23.1. Ma mestrez vad am euz lazet, 23.2. Seiz taol-kontel d'ei 'm euz roêt.

24.1. P'am boa lazet ma mestrez vad, 24.2. Na ouienn pelec'h hi lakad.

25.1. O tont un dra da laret d'in, 25.2. Dre ma oann ken nec'het gant-hi :

26.1. - Na kerz te gant-hi d'ar poull-glaou, 26.2. Ha kuz 'nn ez-hi gant deilou-kraou.

27.1. Me a ieaz neuze d'ar pardon, 27.2. Doue 'ouie ma intantion.

28.1. Me o rankontr ma mestrik mad, 28.2. O kana hag o c'huibanad ;

29.1. O kana hag o c'huibanad, 29.2. Me 'roaz d'ez-han kalonad:

30.1. - Ma mestrez vad a zo lazet, 30.2. Bars ar c'hoad, gant ar forbaned!

31.1. Me vije iwe, penamet

31.2. E-meaz ar c'hoad am euz redet.

32.1. Ma vijac'h bet fidel d'ez-hi, 32.2. C'hui vije lazet koulz ha hi ! 
33.1. Ma mestrik mad, p'hen euz klewet, 33.2. Ter-gwes d'ann douar 'zo koezet ;

34.1. Ter-gwes d'ann douar eo koezet, 34.2. Hag am euz-han bep-gwes savet;

35.1. - Tawet, mestrik, na oelet ket, 35.2. Me ho servijo 'vel bepred ;

36.1. Met n'inn ket ganec'h da gousket, 36.2. Ken 'vomp dimet hag eureujet.

37.1. Sevel 'ra 'tre-z-he prepoziou

37.2. Na diwar-benn ann dimiziou.

38.1. Pa oant dimet hag eureujet, 38.2. Hi poent da vonet da gousket,

39.1. 'C'h antrenn ar c'horf maro en ti, 39.2. Ha seiz sierj koar dira-z-hi ;

40.1. Seiz sierj allum dira-z-hi, 40.2. Unan a oa war bep-gou1i.

41.1. - Savet al lec'h-se, minorez, 41.2. C'hui oc'h euz lazet ho mestrez ;

42.1. C'hui 'c'h euz lazet ho mestrez-vad, 42.2. Tamallet d'ar forbaned er c'hoad!

43.1. Hi friet, pa hen euz klewet, 43.2. 'Meaz he wele a zo zavet ;

44.1. En ur fuzul ez eo kroget, 44.2. En aviz hi lazan eo et :

45.1. Met ar c'horf maro 'n euz laret: 45.2. - Ma fried paour, n' hi lazet ket,

46.1. Met hi leusket da glask hi boed, 46.2. Etre Cavan ha Tonquedec, 47.1. Lec'h na vezo ket anvezet, 47.2. M' tamanto hi c'horf d'hi fec'het!

Kanet gant Janet ar Gall

Plouaret, 1853

\section{Margodig Gillard (Gwerziou II-33)}

1.1. Pan is d'ar stank gant ma dillad,

1.2. Me na sonjen nemet en mad;

1.3. Pa dolis ma linsell et stank,

1.4. Oa paket en-hi ' $n$ inosant!

2.1. Pesketerrienn d-eus ma gwelet,

2.2. Hag a lakas ma c'homerret;

2.3. Hag a lakas ma c'homerret;

2.4. Da gas d'ar prizon d'ann Naonet.

3.1. Ann aotro kapusinn 'lâre,

3.2. Er gador-sarmon pa bigne :

3.3. - En prizon 'n Naonet me 'zo bet,

3.4. Gwalc'h ma c'halon am eûs goelet,

4.1. Gwelet ur plac'hig tric'houec'h vloaz

4.2. Ebarz ar prizon o kana

4.3. Litaniou 'r Werc'hes Varia,

4.4. Hi n' d-eûs ket tri de da vewa!

5.1. Merc'hedigo iaouank, m'ho ped

5.2. Da vont d'ar prizon d'hi gwelet,

5.3. Fourdelizenn ann holl verc'hed,

5.4. Melezour ann itronezed.

6.1. Margodig Gillard 'c'houlenne

6.2. Digant ' $r$ merc'hed iaouank 'n de-se :

6.3. - Merc'hedigo, d'in lavaret

6.4. Petra a-newez 'c'h eûs klewet?

7.1. P'oc'h deut d'am gwelet d'ar prizon,

7.2. Klewet hoc'h-eûs a vinn krouget?

7.3. - Leall, Margodig, n'hon eûs ket

7.4. Klewet lâret 'vijeac'h krouget :

8.1. 'Barz ar sarmon ni a zo bet,

8.2. Hag a eo d'imp gourc'hemennet

8.3. Donet d'ar prizon d'ho kwelet,

8.4. Fourdelizenn ann holl verc'hed:

9.3. Fourdelizenn ann holl verc'hed,

9.4. Melezour ann itronezed.

9.3. - Ewit fourdelizenn n'on ket,

9.4. Melezour d'ac'h, na lârann ket. 
10.1. Merc'hedigo iaouank, m'ho peed

10.2. Karante kloarek n'ho pô ket :

10.3. Dre ma c'hanenn ma bugale, 10.4. Ar c'hloarek 'frike anezhe;

11.1. Mont 'ree war-n-ezhe gant he droad;

11.2. Mill mallos did, kloarek ingrat!

11.3. Merc'hed iaouank, mar am c'hredet,

11.4. Karante kloarek n'hô po ket!

12.1. It d'ann offern, d'ar gousperou,

12.2. A dorr kalz a blanedennou :

12.3. 'Tre ' $n$ offern hag ar gousperou

12.4. Ni gomette hon pec'hejou.

13.1. Merc'hed iaouank, em retiret, 13.2. Me 'well un' gant ar ru 'tonet; 13.3. Me 'well unan 'tont gant ar ru, 13.4. Wit ma glachar, me a zo sur.

14.1. - Na eo ket me eo ann hini, 14.2. Margodig, 'deu d'ho klac'hari;

14.3. Komerret kador, azeet,

14.4. Setu ho setans, hi lennet;

15.1. N'oa ket he setans digorret-mad, 15.2. Ma kouee ' $n$ dour he daoulagad; 15.3. Ma kouee ' $\mathrm{nn}$ dour he daoulagad; 15.4. Hag euz he diou-fran 'kouee ' $r$ gwad!

16.1. - Margodig, d'in-me lavaret 16.2. Na ped bugel hoc'h eûs ganet? 16.3. - Ur menig rouz 'zo 'n prad ma zad, 16.4. Mar gwife komz, hen goar er-fad.

17.1. Me 'm eûs 'dann-han tri inosant, 17.2. Hep ole hag hep badeziant; 17.3. Hag un-all ' $m$ eûs er punz duze, 17.4. Ha seis livr mein euz he goste; 18.1. Ha c'hoaz sav war-c'horre ann dour, 18.2. D' c'houl' digant he dad badeziant; 18.3. D' c'houl' digant he dad badeziant, 18.4. Digant he vamm soulajamant!

19.1. Un-all ' $m$-eûs indann ann treuzou, 19.2. Un tammig teill en he c'henaou; 19.3. Hag un-all indann ann oaled,
19.4. N' oûn penaoz n'oun gant ' $n$ ifern [lonket!...

Chanté par Marie-Josèphe Cado, Plouaret - 1847.

\section{Kervegan hag Ann Tourello (Gwerziou II-24)}

1.1. Kervegan hag ann Tourello

1.2. A zo mignoned a bell ' $z 0$;

2.1. A zo a bell 'zo mignoned,

2.2. Mat war ar gwin hag ar merc'hed.

3.1. Na Kervegan a lavare

3.2. D'ann Tourello, un dez a oe :

4.1. - Me 'c'h a d' vaner ann Dour brema,

4.2. Ewit goulenn ar verc'h hena;

5.1. Ann Tourello a lavare

5.2. Da Gervegan eno neuze :

6.1. - Na ve ket traïtour em andret,

6.1. Ewit-hon eo 'vô goulennet.

7.1. Ann aotrone a voujoure,

7.2. En maner an Dour p'arrue :

8.1. - Boujour ha joa bars ann ti-ma,

8.2. Ar verc'h hena pelec'h ema?

9.1. - Ema duze ' $n$ kambr an dourell,

9.2. Gant-hi diou pe der dimezell.

10.1. Ar verc'h hena, pa d-eûs klewet,

10.2. Gant ' $r$ vinz d'ann traon 'zo diskennet;

11.1. Gant ' $r$ vinz d'ann traon eo diskennet,

11.2. Ha d'he zad e d-eûs lavaret :

12.1. - Diwallet, ma zad, ho pe grêt,

12.2. Rag 'wit Kervegan n'am bô ket;

13.1. Gwell' eo ganen ann Tourello

13.2. Wit Kervegan gant he vado; 
14.1. Ha Kervegan, p'hen eûs klewet, 14.2. D'ann Tourello hen eûs lâret :

15.1. - Deus war da hinkane bihan, 15.2. Ma 'e efomp-ni d'ar gêr buhan.

16.1. En hent-glaz pa int diskennet, 16.2. Kervegan hen eûs lavaret:

17.1. - Diskenn diwar d'hinkane brema, 17.2. Ma c'hoarifomp 'r c'hlewez ama.

18.1. Ann Tourello a lavare

18.2. Da Gervegan, eno, neuze :

19.1. - Me 'wie 'r vad, euz da feson, 19.2. E wiskes dillad ur poultron!

20.1. Diwar he hinkane eo diskennet, 20.2. Nag ewit c'hoari ar fleuret.

21.1. P'oa Kervegan 'c'hoari out-han, 21.2. 'Oa ' $n$ all dreg he geign hoc'h hen

[lac'han.

22.1. Kenta ho d-eûs klewet ar gri,

22.2. 'Oa Lokrenan ha tud he di.

23.1. 'Oa Lokrenan hag he daou vab,

23.2. 'Oa o chaseal er brouz-koad.

24.1. Na Lokrenan a lavare

24.2. D'ann Tourello, eno, neuze :

25.1. - Aotro Doue, a posubl 've 25.2. Ve ann Tourello a vefe!

26.1. Na Lokrenan a lavare

27.1. D'ann Tourello, eno, neuze :

28.1. - Lavar un dra-bennag d'in-me, 28.2. Pa n'hellan klask bêlek d'as ine!

29.1. - Lak' seis offern bars en offrans

29.2. Pa n'hellan disklezria m' c'honsianz;
30.1. Lak' seis offern en Kernitron, 30.2. Honnez 'eo rouanez ann tron;

31.1. Ha laka ter e Sant Efflam, 31.2. Wit $m$ ' inn d'ar baradoz dinam.

32.1. Lokrenan, pa hen eûs klewet, 32.2. War-geign he varc'h a zo pignet;

33.1. War-geign he varc'h ez eo pignet, 33.2. Ha da Lezormel ez eo êt.

34.1. Ar varkizes a lavare

34.2. D'he mates vihan, en noz-se:

35.1. - Lakaët ar bér oc'h an tan, 35.2. Arru ann Tourello d'he goan.

36.1. Na Lokrenan a lavare

36.2. D'ar varkizes, eno, neuze :

37.1. - N' laket ket ar bér oc'h an tan, 37.2. Ann Tourello n' deui ket d'he goan;

38.1. Eman 'I Lew-Drez war he c'heno, 38.2. ' $\mathrm{N}$ aotro Doue d'hen pardono;

39.1. Eman 'I Lew-Drez e-kreiz he wad, 39.2. 'N aotro Doue d'hen joaüssaad.

40.1. Kriz 'vije 'r galon na oelje, 40.2. Bars al Lew-Drez nep a vije,

41.1. 'Welet ' $r$ varkizes leun a ganvo, 42.2. O vriata ar c'horf maro.

42.1. Ha Lokrenan a lavare 42.2. D'ar varkizes, eno, neuze :

43.1. - Na lest Kervegan ganen-me, 43.2. Me 'lakaï barna he vuhe.

Chanté par une servante d'auberge, Au bourg de Plestin - 1864. 